\title{
Dermoscopy for Tick Bite: Reconfirmation of the Usefulness for the Initial Diagnosis
}

\author{
Masahiro Matsuda ${ }^{a}$ Naoki Oiso ${ }^{a}$ Yasuhiro Yano ${ }^{a}$ \\ Akira Kawadab \\ aDepartment of Dermatology, Kinki University Faculty of Medicine, Osakasayama,

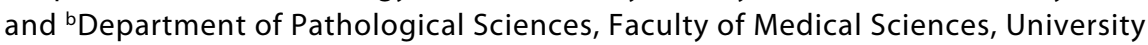 \\ of Fukui, Fukui, Japan
}

\section{Key Words}

Dermoscopy $\cdot$ Tick bite $\cdot$ Japanese spotted fever

\begin{abstract}
Ticks transmit a variety of infectious diseases. Diagnosis requires verification of a tick's presence. Here, we describe a 61-year-old woman bitten by an eight-legged nymphal Amblyomma testudinarium. We re-emphasize the usefulness of dermoscopy for identifying signs of the bite and determining the species of the biting tick.
\end{abstract}

\section{Introduction}

Ticks are vectors of a variety of bacterial and protozoal diseases. It is important to check for the presence of a tick bite, because immediate and accurate treatment may reduce the morbidity and mortality of tick-borne diseases [1]. By dermoscopy, we have previously detected a lesion caused by a tick bite and the tick's lost hypostome in a patient [2]. Dermoscopy can easily detect the six-legged larval and eight-legged nymphal forms $[2,3]$. Here, we describe another case of a tick bite to re-emphasize the usefulness of dermoscopy for making an immediate and accurate diagnosis.

\section{Case Report}

On May 9, 2010, a 61-year-old woman, living in a rural area of Tondabayashi, Osaka, Japan, noticed a papule-like lesion on the right lateral abdomen. On May 13, she discovered that it was a tick and picked it out. On May 15, the patient visited us, because a slightly pruritic eruption had developed on the bite lesion. She often did garden chores. Physical examination revealed a slightly pruritic erythema, $10 \mathrm{~mm}$ in size, on the right lateral abdomen (fig. 1a). Dermoscopic findings at a magnification of $\times 25$ 
showed a round lesion, $0.1 \mathrm{~mm}$ in size, in the center of the erythema (fig. 1b), and the tick without its hypostome (fig. 1c). The tick had a pair of eyes at both sides of the scutum, although the focus of the figure was insufficient to indicate with arrows (fig. 1d). The bite lesion, including the erythema, was locally excised. Hematoxylin and eosin staining showed superficial perivascular dermatitis with the infiltration of eosinophils, lymphocytes, and histiocytes, but no sign of the hypostome. Using dermoscopy, the tick was confirmed to be an eight-legged nymphal Amblyomma testudinarium, which sometimes carries Rickettsia tamurae $[4,5]$. The bite episode caused the patient no discomfort except from the pruritic erythema for 1 month.

\section{Discussion}

In the previous $[2,3]$ and present cases, dermoscopy proved useful for detecting and examining the bite lesion and the captured tick. Dermoscopy can determine whether or not the tick has been completely removed [6], and is useful for detecting and removing the tiny six-legged larval form [7]. Moreover, dermoscopy also allows for easy initial classification of the species of the tick, which will determine the strategy for medical treatment.

During the past 5 years, most of the tick bites seen at our outpatient clinic in the Kansai region have been caused by A. testudinarium, Haemaphysalis flava, and Haemaphysalis longicornis [3]. H. flava and H. longicornis may transmit Rickettsia japonica, the pathologic organism of Japanese spotted fever $[8,9]$. The occurrence of Japanese spotted fever is not rare in the Kansai region [10,11]. Dermoscopy can easily identify the species of the biting tick and is useful for making a preliminary diagnosis in cases of tick bites. 

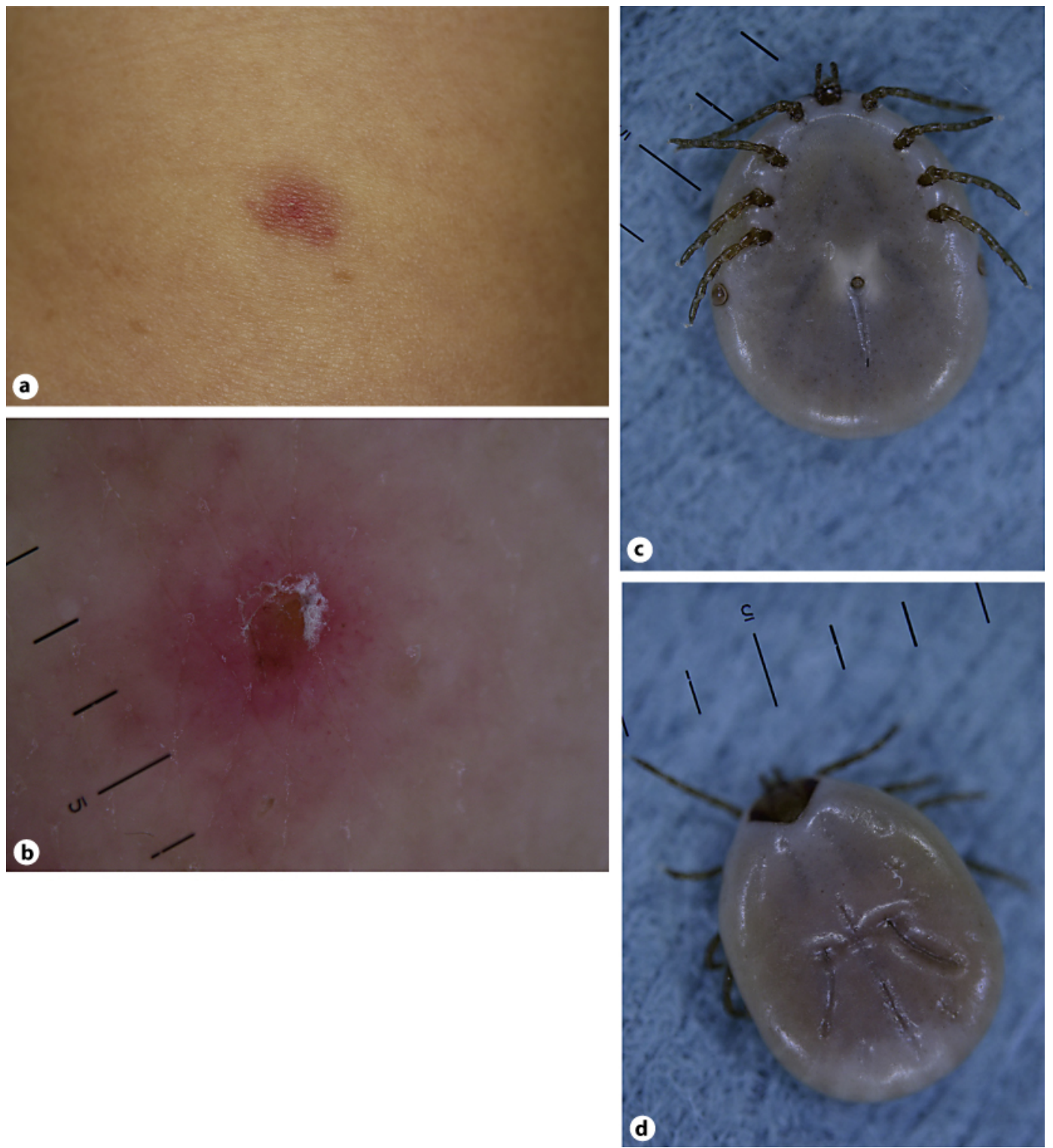

Fig. 1. a Clinical image of the bite lesion on the right lateral abdomen. $\mathbf{b}$ The dermoscopic findings revealed a round lesion, $0.1 \mathrm{~mm}$ in size, on the center of the erythema. c The tick without its hypostome (original magnification $\times 25$ ). $\mathbf{d}$ The dorsal aspect of the tick (original magnification $\times 25$ ).

\section{References}

1 Bratton RL, Corey R: Tick-borne disease. Am Fam Physician 2005;71:2323-2330.

2 Oiso N, Kawara S, Yano Y, Kawada A: Diagnostic effectiveness of dermoscopy for tick bite. J Eur Acad Dermatol Venereol 2010;24:231-232.

-3 Oiso N, Nakano A, Yano Y, Kawada A: The diagnostic usefulness of dermoscopy for identifying six-legged larval ticks. Ticks Tick Borne Dis 2010;1:197-198.

-4 Fournier PE, Takada N, Fujita H, Raoult D: Rickettsia tamurae sp. nov., isolated from Amblyomma testudinarium ticks. Int J Syst Evol Microbiol 2006;56:1673-1675. 
5 Imaoka K, Kaneko S, Tabara K, Kusatake K, Morita E: The first human case of Rickettsia tamurae infection in Japan. Case Rep Dermatol 2011;3:68-73.

6 Zalaudek I, Giacomel J, Cabo H, Di Stefani A, Ferrara G, Hofmann-Wellenhof R, Malvehy J, Puig S, Stolz W, Argenziano G: Entodermoscopy: a new tool for diagnosing skin infections and infestations. Dermatology 2008;216:14-23.

7 Rallis E: Dermoscopy of tick bite. Scientific World Journal 2010;10:1705-1706.

8 Uchida T, Yan Y, Kitaoka S: Detection of Rickettsia japonica in Haemaphysalis longicornis ticks by restriction fragment length polymorphism of PCR product. J Clin Microbiol 1995;33:824-828.

9 Fournier PE, Fujita H, Takada N, Raoult D: Genetic identification of rickettsiae isolated from ticks in Japan. J Clin Microbiol 2002;40:2176-2181.

10 Hashimoto S, Kawado M, Murakami Y, Izumida M, Ohta A, TadaY, Shigematsu M, Yasui Y, Taniguchi K, Nagai M: Epidemics of vector-borne diseases observed in infectious disease surveillance in Japan, 2000-2005. J Epidemiol 2007;17(suppl):S48-S55.

11 Kondo M, Nishii M, Gabazza EC, Kurokawa I, Akachi S: Nine cases of Japan spotted fever diagnosed at our hospital in 2008. Int J Dermatol 2010;49:430-434. 